\title{
Determinantes sociales de salud y necesidad educativa sobre infecciones de transmisión sexual en adolescentes embarazadas
}

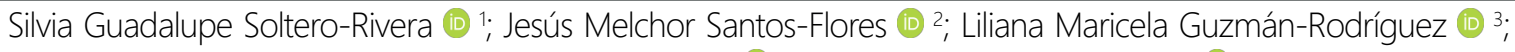

Juana Mercedes Gutiérrez Valverde (iD 4; Milton Carlos Guevara-Valtier (i) 5*

\section{RESUMEN}

Introducción: En los últimos años ha ocurrido un incremento y una mayor precocidad del inicio sexual provocando un aumento de la incidencia de embarazo adolescente, su prevalencia varía a nivel mundial. Las infecciones de transmisión sexual son reconocidas como un gran problema de salud pública y puede ser prevenible. Objetivo: Conocer los determinantes sociales de salud presentes para un mayor riesgo de un embarazo a temprana edad e identificar la necesidad educativa durante la etapa de adolescencia sobre conocimiento de las Infecciones de Transmisión Sexual. Metodología: Diseño descriptivo transversal, la muestra fue de 60 adolescentes embarazadas de 10 a 19 años de edad, se utilizó un la Escala sobre conocimiento del virus de la inmunodeficiencia humana y otras infecciones de transmisión sexual con Alfa de Cronbach de .66 a .88. Resultados: Se encontró que un 38.3\% de la muestra encuestada tiene antecedentes de embarazos adolescentes en la familia. El 46\% desconoce los métodos de transmisión del virus de inmunodeficiencia humana y el 50\% desconoce la transmisión del virus de papiloma humano, un 50\% desconoce en general sobre las infecciones de transmisión sexual. Conclusión: Los principales determinantes sociales en salud encontrados son los factores familiares como el tipo de familia (biparental), antecedentes de embarazos adolescentes en la familia, en el factor sexual y reproductivo como el inicio de la primera relación sexual a temprana edad, tener más de dos parejas sexuales, y el uso previo de métodos anticonceptivos, y la falta de conocimiento.

Palabras clave: Conducta del Adolescente; Conocimiento; Enfermedades de transmisión sexual (DeCS).

1. Doctorado en Ciencias de Enfermería. Universidad Autónoma de Nuevo León. Monterrey, Nuevo León, México.

2. Maestría en Ciencias de Enfermería, Universidad Autónoma de Nuevo León. Monterrey, Nuevo León, México.

3. Doctorado en Educación. Universidad Autónoma de Nuevo León. Monterrey, Nuevo León, México.

4. Doctorado en Enfermería. Universidad Autónoma de Nuevo León. Monterrey, Nuevo León, México.

5. Doctorado en Educación. Universidad Autónoma de Nuevo León. Monterrey, Nuevo León, México.

Recibido: $04 / 01 / 2020$

Aceptado: 22/06/2020

*Autor para correspondencia: carlos_valtier7@hotmail.com

Copyright $\odot 2020$ SANUS

Artículo de acceso abierto distribuido

bajo Licencia Creative Commons

\section{c) (i) $९$

\section{Cómo citar este artículo}

Soltero-Rivera SG, Santos-Flores JM, Guzmán-Rodríguez LM, Gutiérrez-Valverde JM, Guevara-Valtier MC. Determinantes sociales de salud y necesidad educativa sobre infecciones de transmisión sexual en adolescentes embarazadas. SANUS. 2020; (14): 1-11.[Acceso__ _ _ ]; Disponible en: 


\title{
Social health determinants and education need about sexua- lly transmitted infections in pregnant adolescents
}

\begin{abstract}
Introduction: In recent years there has been an increase and a greater precocity of sexual initiation causing an increase in the incidence of adolescent pregnancy, but its prevalence varies worldwide. Sexually Transmitted Infections are recognized as a major public health problem but can be prevented. Objective: Know the Social Health Determinants present for a higher risk of early pregnancy and identify education need during the adolescence stage regarding knowledge of Sexually Transmitted Infections. Methodology: A cross-sectional descriptive design was used. The sample was comprised by 60 pregnant adolescents ranging in age from 10 to 19 years. A scale regarding knowledge of the human immunodeficiency virus and other sexually transmitted infections was used with a Cronbach's Alpha ranging from .66 to .88. Results: It was found that $38.3 \%$ of the surveyed sample has a history of adolescent pregnancies in the family. Forty percent of them were unaware of the transmission methods of the Human Immunodeficiency Virus and 50\% are unaware of the transmission of the Human Papilloma Virus. In general, 50\% are unaware of sexually transmitted infections. Conclusion: The main social health determinants found were family factors such as family type (two-parent family) and history of adolescent pregnancies in the family; regarding sexual and reproductive factors there is the beginning of the first sexual relation at an early age, having more than two sexual partners, previous use of contraceptive methods, and lack of knowledge.
\end{abstract}

Key words: Adolescent; Knowledge; Sexually Transmitted Diseases (DeCS). 


\title{
Determinantes da saúde social e necessidade de educação sobre infecções sexualmente transmissíveis em adolescentes grávidas
}

\begin{abstract}
ABSTRATO
Introdução: Nos últimos anos, houve um aumento e uma maior precocidade de iniciação sexual, causando um aumento na incidência de gravidez na adolescência, mas sua prevalência varia em todo o mundo. As infecções sexualmente transmissíveis são reconhecidas como um importante problema de saúde pública, mas podem ser evitadas. Objetivo: Conheça os determinantes sociais da saúde que apresentam maior risco de gravidez precoce e identificar a necessidade de educação durante a adolescência em relação ao conhecimento de infecções sexualmente transmissíveis. Metodologia: Foi utilizado um desenho descritivo transversal. A amostra foi composta por 60 adolescentes grávidas com idade entre 10 e 19 anos. Uma escala referente ao conhecimento do vírus da imunodeficiência humana e outras infecções sexualmente transmissíveis foi usada com um alfa de Cronbach variando de 0,66 a 0,88. Resultados: Verificou-se que 38,3\% da amostra pesquisada têm histórico de gravidez na adolescência na família. Quarenta por cento de lês desconheciam os métodos de transmissão do vírus de imunodeficiência humana e 50\% desconheciam a transmissão do vírus do papiloma humano. Em geral, 50\% desconhecem infecções sexualmente transmissíveis. Conclusões: Os principais determinantes da saúde social encontrados foram fatores familiares, como tipo de família (biparental) e história de gravidez na adolescência na família; em relação aos fatores sexuais e reprodutivos, há o início da primeira relação sexual em tenra idade, com mais de dois parceiros sexuais, uso prévio de métodos contraceptivos e falta de conhecimento.
\end{abstract}

Palavras chave: Adolescente; Conhecimento; Doenças Sexualmente Transmissíveis (DeCS). 


\section{INTRODUCCIÓN}

Según la Organización Mundial de la Salud (OMS) (1) a nivel mundial un gran número de nacimientos, aproximadamente un $11 \%$, se producen en adolescentes en su mayoría de países de bajo y mediano ingreso; antes de los 18 años de edad cerca del 30\% contraen matrimonio, a los 15 años el 14\%, así mismo, la tasa de natalidad a nivel mundial en este grupo de edad es de 4.9 por cada $100{ }^{\text {(2). }}$. De acuerdo a la literatura en países de Latinoamérica, cerca de una cuarta parte de los nacidos vivos son hijos de madres adolescentes ${ }^{(3)}$, en este contexto México ocupa el primer lugar de embarazos en adolescentes con una tasa de 77 nacimientos por cada mil jóvenes de 15 a 19 años. Según el Instituto Nacional de Estadística y Geografía (INEGI) ${ }^{(4)}$ Nuevo León es el décimo lugar con 14,736 embarazos en adolescentes en el país; el primer lugar con 53,105 lo tiene el Estado de México.

Una de las etapas del desarrollo humano con importantes cambios a nivel social, psicológico y biológico es la adolescencia, durante este periodo aumentan las conductas de riesgo que llevan a consecuencias peligrosas y nocivas como indicó Rosabal (5) a partir de la perspectiva de que jóvenes y adolescentes emergen de forma importante en temas tan vitales como la salud reproductiva y sexual por lo que en la etapa de maduración sexual existe una búsqueda temprana de relaciones sexuales por lo que se exponen a diversos problemas de salud (6).

La Secretaria de Salud de Nuevo León (7) menciona que las Infecciones de Transmisión Sexual (ITS) "son infecciones que se adquieren al tener relaciones sexuales con una persona infectada", también refiere que la mayoría afectan a hombres tanto como a mujeres, pero en muchos casos los problemas de salud suelen ser más graves en las mujeres, un ejemplo es la infección por el Virus del Papiloma Humano (VPH), por otro lado si una mujer embarazada padece de ITS existe el riesgo de que el producto tenga graves problemas de salud ${ }^{(8)}$.

Según la literatura existe una alta incidencia de ITS que han ido en aumento en la población adolescente, posiblemente estas conductas de riesgo sean producto de ello al igual de la prevalencia de embarazos no deseados en esta población; otros estudios señalan que la educación sería una medida que puede mejorar la prevención de comportamientos sexuales de riesgo. Según el Instituto Nacional de las Mujeres ${ }^{\left({ }^{9}\right)}$ existe cierto porcentaje de población adolescente (15\% hombres y $33 \%$ de las mujeres) que no usa métodos anticonceptivos en su primera relación.

Vásquez ${ }^{(10)}$ refiere que existen cambios físicos a nivel biológico en la adolescencia, algunos cambios son el desarrollo de los caracteres sexuales secundarios, maduración en las gónadas y una estimulación en la producción de las hormonas asociadas a cambios a nivel conductual. También menciona que el adolescente es vulnerable y asu- me ciertos comportamientos de riesgo, como lo es el inicio de la actividad sexual temprana. Refiere que los adolescentes pueden ser fértiles aproximadamente cuatro o cinco años antes de su maduración emocional, por lo que el inicio sexual a temprana edad aumenta el riesgo de embarazos no deseados, ITS y prácticas como el aborto ${ }^{(10)}$.

La Organización de las Naciones Unidas para la Educación, la Ciencia y la Cultura (UNESCO) (11) refiere que, a pesar de la evidencia a favor de los beneficios de una educación integral en sexualidad de excelente calidad, existen pocos jóvenes que reciben una preparación para la vida que los capacite para llevar el control y tomar decisiones conscientes acerca de su sexualidad y sus relaciones de manera responsable.

Es importante que los temas de salud sexual y sexualidad sean puestos en la práctica educativa, impartidos y explicados en aulas escolares por profesionales del ramo y/o docentes capacitados desde etapas tempranas, asimismo involucrar a la familia y a los servicios de salud podría garantizar un mayor impacto en la promoción de la salud sexual y reproductiva ${ }^{(10)}$. La falta de información acerca de la salud sexual lleva a los jóvenes a un comportamiento sexual de riesgo y embarazos no deseados, una de las causas predisponentes es la falta de acceso a la educación o a los servicios de salud sexual y como consecuencia uso de manera inadecuada de métodos de planificación familiar o simplemente tienen desconocimiento de los mismos (12).

El padecer una ITS durante el embarazo puede ser perjudicial para el producto. La gonorrea y clamidia pueden provocar problemas de salud, como infecciones oculares y pulmonares en los recién nacidos. La Síflis puede producir abortos espontáneos o el nacimiento de un producto obitado, y en cuanto al VIH, este puede ser contraído por el feto en el medio placentario a través del cordón umbilical ${ }^{(13)}$. Por lo anterior, el objetivo general del presente estudio fue conocer los determinantes sociales de salud presentes para un mayor riesgo de un embarazo a temprana edad e identificar la necesidad educativa durante la adolescencia sobre conocimiento de las ITS.

\section{METODOLOGÍA}

El diseño del estudio fue descriptivo transversal ${ }^{(14,15)}$. La población la conformaron adolescentes embarazadas con edades de entre 10 a 19 años que asistieron a la Unidad Médica Familiar en Monterrey, Nuevo León. El muestreo fue por conveniencia, obteniendo una muestra de 60 adolescentes gestantes con edades entre 10 a 19 años.

Se utilizó la Entrevista de Percepción de Carga del Cuidado de Zarit (EPCCZ) en español de Barreto ${ }^{(16)}$ con 13 ítems que indagan factores sociodemográficos, familiares y de tipo sexual/ reproductivo que se asocian al embarazo en adolescentes utilizados para describir los factores que influyen en la gestación.

Se hizo uso de la Escala sobre conocimiento del $\mathrm{VIH}$ y otras ITS (ECVIH, ITS) de Espada y colaboradores ${ }^{(17)}$, cuenta 
Tabla 1. Factores Sociodemográficos de adolescentes gestantes de una Unidad Médico Familiar

\begin{tabular}{|l|c|c|}
\hline & $\boldsymbol{f}$ & \% \\
\hline Grado de instrucción & & 1.7 \\
Ninguna & 1 & 3.3 \\
Primaria & 2 & 38.3 \\
Secundaria & 23 & 56.7 \\
Superior & 34 & \\
\hline Ocupación & 27 & 45 \\
Ama de casa & 8 & 13.3 \\
Estudiante & 25 & 41.7 \\
Trabaja & & \\
\hline Estado civil & 10 & 16.7 \\
Soltera & 35 & 58.3 \\
Casada & 15 & 25 \\
Conviviente & \multicolumn{2}{|c|}{} \\
\hline
\end{tabular}

Fuente: $E P C C Z, f=$ frecuencia, \%= porcentaje

con 40 preguntas relacionadas sobre el conocimiento $\mathrm{VIH}$ y otras infecciones de transmisión sexual. Esta escala a su vez integra subescalas, la primera se denomina, (SEVIH-ITS) sobre conocimiento del VIH y otras ITS, la segunda (SEMT-VIH) mecanismos de transmisión del VIH, la tercera (SE-MPPVIH) subescala mecanismos de protección para prevenir la infección por $\mathrm{VIH}$, la cuarta (SE-MAVIH) subescala mecanismo de acción del VIH en el organismo y tratamiento del $\mathrm{VIH}$, la quinta (SE-CGGS) subescala características generales de la gonorrea y síflis, la sexta subescala (SE-CHBVG) conocimiento respecto a la hepatitis $\mathrm{B}$ y verrugas genitales (Dimensiones: conocimiento transmisión VIH, otras ITS, conocimiento general VIH, preservativo y Prevención VIH ) (Alfa de Cronbach que de .66 a .88). Para la realización de este estudio se contó con aprobación de una institución educativa y de salud implicada en la colecta de datos.

Para la recolección de datos se contactó y explicó a los padres, tutores o representantes legales y a las adolescentes sobre el objetivo del estudio y la participación de las adolescentes en el mismo; realizado lo anterior, se formalizó la invitación y a quienes la aceptaron se les proporciono el consentimiento y asentimiento para lectura y firma; se les brindaron los cuestionarios invirtiendo entre 10 a 15 minutos en contestar las preguntas, con el fin de mantener la privacidad e intimidad de las participantes se contó con un consultorio de medicina preventiva en el que estuvo presente la adolescente y el investigador. Los padres, tutores, o representantes legales esperaban en una sala contigua.

Se les solicitó a las participantes ser honestas, contestar acorde a su realidad, las dudas que surgieron fueron aclaradas. Se respetó el derecho a abandonar el estudio, hecho que no se presentó. Aunado a lo anterior se comunicó que los resultados derivados del presente estudio se tratarían de manera anónima y confidencial, al respecto las participantes entregaban su cuestionario en un sobre cerrado y sellado. Este estudio se apegó al Reglamento de la Ley General de Salud en Materia de Investigación para la Salud (18), se contó con la aprobación del Comité de Investigación y Ética en Investigación con número de registro 015-02018.

Se utilizó el programa estadístico IBM SPSS, versión Statistics 23.0, para dar respuesta al objetivo se emplearon frecuencias, porcentajes y medidas de tendencia central.

\section{RESULTADOS}

Participaron 60 adolescentes embarazadas. En la tabla 1 se presentan los factores sociodemográficos, la media de edad fue de 17.8 años ( $D E=1.20)$, así mismo se encontró que $56.7 \%$ de las adolescentes tenían un grado de instrucción superior y la mayoría se encontraban en estado civil casada (58.3\%).

En la tabla 2 se incluyen los factores familiares, se observa que la mayoría lleva una buena relación en sus hogares con $73.3 \%$, la mayoría (61.7\%) no tuvo antecedentes de embarazos adolescentes en la familia y se encontró que $75 \%$ vivía con ambos padres antes del embarazo.

La tabla 3 muestra el factor sexual y reproductivo, 90\% inició su primera relación sexual entre los 15 y los 19 años. El 65\% utilizó métodos anticonceptivos antes del embarazo. Los datos indican que $48.3 \%$ de las adolescentes son primigestas, $38.3 \%$ es su segunda gesta y $90 \%$ no tuvo antecedentes de aborto.

La tabla 4 contempla en la subescala SEVIH-ITS sobre conocimiento del VIH y otras ITS del EPCCZ, las adolescentes contestaron en su mayoría como verdadero en las afirmaciones: "El SIDA es causado por un virus Ilamado VIH" (86.7\%) y "La principal vía es transmisión del VIH es a través de las relaciones sexuales" (90\%). 
Tabla 2. Factores Familiares de adolescentes gestantes de una Unidad Médico Familiar

\begin{tabular}{|c|c|c|}
\hline & $f$ & $\%$ \\
\hline $\begin{array}{l}\text { Tipo de familia del adolescente } \\
\text { Monoparental } \\
\text { Biparental }\end{array}$ & $\begin{array}{l}15 \\
45\end{array}$ & $\begin{array}{l}25 \\
75\end{array}$ \\
\hline $\begin{array}{l}\text { Antecedentes de embarazos adolescentes en la familia } \\
\text { Si } \\
\text { No }\end{array}$ & $\begin{array}{l}23 \\
37\end{array}$ & $\begin{array}{l}38.3 \\
61.7\end{array}$ \\
\hline $\begin{array}{l}\text { Relación del adolescente con sus padres } \\
\text { Buena } \\
\text { Regular } \\
\text { Mala }\end{array}$ & $\begin{array}{c}44 \\
16 \\
0\end{array}$ & $\begin{array}{c}73.3 \\
26.7 \\
0\end{array}$ \\
\hline $\begin{array}{l}\text { Persona con quien vivía antes de su embarazo } \\
\text { Padre } \\
\text { Madre } \\
\text { Ambos } \\
\text { Solo } \\
\text { Otro familiar }\end{array}$ & $\begin{array}{c}3 \\
9 \\
45 \\
2 \\
1\end{array}$ & $\begin{array}{c}5 \\
15 \\
75 \\
3.3 \\
1.7\end{array}$ \\
\hline
\end{tabular}

Fuente: $\mathrm{EPCCZ}, f=$ Frecuencia; \% = Porcentaje

En la segunda subescala SEMT-VIH mecanismos de transmisión del $\mathrm{VIH}$, contestaron mayormente como verdadero al "Riesgo de contraer VIH por jeringas" con 98.3\%, "El VIH afecta el sistema inmunológico humano" con $88.3 \%$. En "El virus del SIDA afecta a todas las células del organismo humano" 90\% de la población encuestada considera es verdadera. Mientras que el reactivo "EI VIH se transmite por el aire" fue contestada como falsa por $96.7 \%$.

En la tercera SE-MPPVIH subescala mecanismos de protección para prevenir la infección por VIH. Las siguientes afirmaciones predominaron con la respuesta de "Falso" "El anillo vaginal o el DIU son métodos eficaces para evitar el SIDA" (85\%). "Las píldoras anticonceptivas son eficaces para prevenir la transmisión del VIH en las relaciones sexuales" 86.7\%, "Abrazar y besar en la mejilla a una persona con el virus implica riesgo de transmisión del VIH" (90\%). "Practicar el coito interrumpiendo la eyaculación es una forma segura de practicar sexo de riesgo de infección por VIH" (80\%).

En la cuarta SE-MAVIH subescala mecanismo de acción del $\mathrm{VIH}$ en el organismo y tratamiento del $\mathrm{VIH}$, las afirmaciones que predominaron como verdaderas fueron: "La prueba de detección del VIH se suele realizar mediante un análisis de sangre" (98.3\%) y "La gonorrea y la clamidia son dos enfermedades de transmisión sexual comunes" con 90\%. Se detectó que una necesidad educativa de importancia corresponde a la pregunta "Cuando la pareja tiene gonorrea hay que tratarla" 55\% respondió falso.

En la quinta SE-CGGS subescala características generales de la gonorrea y síflis la afirmación "La gonorrea o gonococia se curan solas en la mayoría de los casos" es considerada como falsa (81.7\%) y "No es conveniente mantener relaciones sexuales si se tiene una infección por herpes genital" respondieron con 93.3\%.

En la sexta subescala SE-CHBVG conocimiento respecto a la Hepatitis B y verrugas genitales la afirmación "Las verrugas genitales se contagian durante las relaciones sexuales" es considerada como verdadera por un $83.35 \%$. En de la séptima subescala Conocimiento sobre el VPH la afirmación "Hay vacunas para el VPH" fue considerada como verdadera con $88.3 \%$.

\section{DISCUSIÓN}

El presente estudio se incorpora a la literatura limitada sobre los determinantes sociales de salud presentes para un mayor riesgo de un embarazo a temprana edad y la identificación del nivel de conocimiento sobre las ITS. En relación a los resultados del estudio, más de la mitad de las participantes presentaban un grado de estudio superior, en su mayoría se reportaban como casadas y tenían un empleo, en comparación con otros estudios donde la mayor proporción de adolescentes habían estudiado hasta el nivel de secundaria, se encontraban actualmente solteras y se dedicaban al hogar entre 43 y el $97.1 \%{ }^{(19,21,22)}$.

Las diferencias podrían deberse al área geográfica en donde se realizaron los estudios, debido a que los estudios efectuados por Rodríguez ${ }^{(19)}$, Silva, et al., (21) y Contreras, et al., (22) fueron en zonas del sur de México y países de América del Sur en donde las costumbres y culturas son distintas en cuanto al matrimonio y la educación (21).

En cuanto a los factores familiares, $75 \%$ vivían en familia bi- 
Tabla 3. Factor Sexual y Reproductivo de adolescentes gestantes de una Unidad Médico Familiar

\begin{tabular}{|c|c|c|}
\hline & $f$ & $\%$ \\
\hline $\begin{array}{l}\text { Inicio de la primera relación sexual } \\
10 \text { a } 14 \text { años } \\
15 \text { a } 19 \text { años }\end{array}$ & $\begin{array}{c}6 \\
54\end{array}$ & $\begin{array}{l}10 \\
90\end{array}$ \\
\hline $\begin{array}{l}\text { Número de parejas sexuales } \\
1 \\
\text { Más de } 2\end{array}$ & $\begin{array}{l}28 \\
32\end{array}$ & $\begin{array}{l}46.7 \\
53.3\end{array}$ \\
\hline $\begin{array}{l}\text { Número de gestaciones } \\
\text { Primigesta } \\
\text { Segundigesta } \\
\text { Multigesta }\end{array}$ & $\begin{array}{c}29 \\
23 \\
8\end{array}$ & $\begin{array}{l}48.3 \\
38.3 \\
13.3\end{array}$ \\
\hline $\begin{array}{l}\text { Antecedentes de aborto } \\
\text { Si } \\
\text { No }\end{array}$ & $\begin{array}{c}6 \\
54\end{array}$ & $\begin{array}{l}10 \\
90\end{array}$ \\
\hline $\begin{array}{l}\text { Usos previos de métodos anticonceptivos antes de un embarazo } \\
\text { Si } \\
\text { No }\end{array}$ & $\begin{array}{l}39 \\
21\end{array}$ & $\begin{array}{l}65 \\
35\end{array}$ \\
\hline
\end{tabular}

Fuente: $E P C C Z, f=$ Frecuencia; \% = Porcentaje

$\boldsymbol{n}=60$

parental, diferentes resultados encontrados por Rodríguez ${ }^{(19)}$ donde reportó que más de la mitad de las adolescentes vivían en familia monoparental, es por ello que en su estudio la mayoría eran adolescentes solteras. De acuerdo al factor sexual y reproductivo, en el presente estudio el $90 \%$ tuvo su primera relación sexual en edad comprendidas de 15 a 19 años, en cuanto a otros estudios estos hallazgos son similares a los de Rodríguez ${ }^{(19)}$ pero diferentes a los de Silva ${ }^{(21)}$ donde encontró que en la mayoría de las adolescentes la primera relación sexual fue de 14 a 16 años de edad; en el presente estudio más de la mitad tuvieron dos parejas sexuales mientras que Rodríguez ${ }^{(19)}$ encontró que 53\% tuvo una sola pareja sexual, así mismo se encontró que la mayoría eran primigestas, similar a lo encontrado por Rodríguez ${ }^{(19)}$.

Una posible explicación con relación a los factores tanto familiares como sexuales y reproductivos es que las conductas de riesgo en adolescentes son determinantes sociales en la salud y las principales causas responsables del embarazo precoz e ITS independientemente de la nación o zona geográfica (20).

De acuerdo al nivel de conocimientos, en el presente estudio se encontró que $50 \%$ tiene un conocimiento deficiente sobre las ITS, similar a lo encontrado por otros estudios donde el nivel de conocimientos fue regular, en su mayoría (80\%) no cuentan con conocimientos adecuados sobre las ITS (21, 22, 23). Si bien el VIH, debido a las campañas de divulgación es una enfermedad bien conocida tanto por las adolescentes como la población en general, sin embargo, otras ITS son menos conocidas, tanto los nombres de las ITS como sus síntomas, y mecanismos de contagio (28).

\section{CONCLUSIONES}

De acuerdo con los resultados se puede afirmar con cautela que los principales determinantes sociales en salud entendidos como las circunstancias en que las personas nacen, crecen, viven, trabajan y envejecen, incluido el sistema de salud, en el presente estudio fueron los factores familiares como el tipo de familia (biparental), antecedentes de embarazos adolescentes en la familia, en el factor sexual y reproductivo como el inicio de la primera relación sexual a temprana edad, tener más de dos parejas sexuales, y el uso previo de métodos anticonceptivos, en cuanto a los conocimientos tener desconocimiento de las ITS. La falta de conocimiento de la población participante respecto a las ITS es un indicador a tomarse en cuenta al momento de planificar y llevar a cabo intervenciones en materia de educación sexual en la población adolescente y con ello coadyuvar en la prevención de este problema de salud pública.

\section{CONFLICTO DE INTERESES}

No existen conflictos de intereses.

\section{FINANCIAMIENTO}

No existió. 
Tabla 4. Estadística descriptiva de la Escala sobre Conocimiento del VIH y otras ITS de adolescentes gestantes de una Unidad Médico Familiar

\begin{tabular}{|c|c|c|c|c|}
\hline \multirow{2}{*}{ Subescalas } & \multicolumn{2}{|c|}{ Verdadero } & \multicolumn{2}{|c|}{ Falso } \\
\hline & $f$ & $\%$ & $f$ & $\%$ \\
\hline \multicolumn{5}{|l|}{ 1) Diferencias entre VIH - SIDA } \\
\hline EI SIDA es causado por VIH & 52 & 86.7 & 8 & 13.3 \\
\hline Existe diferencia entre $\mathrm{VIH}$ y SIDA & 33 & 55 & 27 & 45 \\
\hline Principal vía de transmisión del VIH & 54 & 90 & 6 & 10 \\
\hline Los animales domésticos transmiten VIH/ SIDA & 6 & 10 & 54 & 90 \\
\hline Una mujer embarazada puede transmitir el VIH al bebé & 50 & 83.3 & 10 & 16.7 \\
\hline Las personas con VIH pueden transmitir el SIDA si no están enfermas & 35 & 58.3 & 25 & 41.7 \\
\hline El VIH se transmite por secreciones y sangre & 56 & 93.3 & 4 & 6.7 \\
\hline \multicolumn{5}{|l|}{ 2) Mecanismos de transmisión del VIH } \\
\hline El VIH se transmite por el aire & 2 & 3.3 & 58 & 96.7 \\
\hline Es peligrosos compartir alimentos con personas con VIH/ SIDA & 14 & 23.3 & 46 & 76.7 \\
\hline Lavar la ropa con la de una persona con VIH/ SIDA & 13 & 21.7 & 47 & 78.3 \\
\hline Los mosquitos trasmiten VIH/ SIDA & 19 & 31.7 & 41 & 68.3 \\
\hline Riesgo de contraer VIH por jeringas & 59 & 98.3 & 1 & 1.7 \\
\hline El VIH afecta el sistema inmunológico & 53 & 88.3 & 7 & 11.7 \\
\hline EI VIH/ SIDA afecta todas células del organismo & 54 & 90.0 & 6 & 10.0 \\
\hline \multicolumn{5}{|l|}{ 3) Mecanismos de protección para prevenir la infección por VIH } \\
\hline El periodo ventana es el tiempo en producir anticuerpos & 46 & 76.7 & 14 & 23.3 \\
\hline El anillo vaginal o DIU evitan el SIDA & 9 & 15.0 & 51 & 85.0 \\
\hline Las píldoras previenen el VIH & 8 & 13.3 & 52 & 86.7 \\
\hline El preservativo femenino es eficaz para evitar el VIH/SIDA & 45 & 75.0 & 15 & 25.0 \\
\hline Un beso húmedo es riesgo de transmisión del VIH & 26 & 43.3 & 34 & 56.7 \\
\hline Abrazar y besar implica riesgo & 6 & 10.0 & 54 & 90.0 \\
\hline El coito interrumpido evita la trasmisión de VIH & 12 & 20.0 & 48 & 80.0 \\
\hline \multicolumn{5}{|c|}{ 4) Mecanismo de acción del VIH en el organismo y tratamiento del VIH } \\
\hline La detección de VIH se realiza por prueba de sangre. & 59 & 98.3 & 1 & 1.7 \\
\hline Es posible saber si tiene VIH al día siguiente del riesgo & 28 & 46.7 & 32 & 53.3 \\
\hline Los tratamientos reducen la cantidad de $\mathrm{VIH}$ & 41 & 68.3 & 19 & 31.7 \\
\hline Existe una vacuna contra el VIH & 30 & 50.0 & 30 & 50.0 \\
\hline Las infecciones aparecen por el debilitamiento del sistema inmune & 42 & 70.0 & 18 & 30.0 \\
\hline La gonorrea y clamidia son ITS & 54 & 90.0 & 6 & 10.0 \\
\hline Cuando la pareja tiene gonorrea hay que tratarla & 27 & 45.0 & 33 & 55.0 \\
\hline \multicolumn{5}{|l|}{ 5) Características generales de la gonorrea y sífilis } \\
\hline La gonorrea o gonococia puede pasar sin síntomas a la mujer & 30 & 50.0 & 30 & 50.0 \\
\hline La gonorrea o gonococia se curan solas & 11 & 18.3 & 49 & 81.7 \\
\hline La síflis es una enfermedad desaparecida & 20 & 33.3 & 40 & 66.7 \\
\hline La sífilis puede dejar lesiones permanentes & 45 & 75 & 15 & 25 \\
\hline
\end{tabular}




\begin{tabular}{|l|l|l|l|l|}
\hline El contagio de la síflis es muy difícil & 26 & 43.3 & 34 & 56.7 \\
\hline El contagio por herpes genital solo da síntomas en dicha zona & 42 & 70.0 & 18 & 30.0 \\
\hline No mantener relaciones si se tiene herpes genital & 56 & 93.3 & 4 & 6.7 \\
\hline
\end{tabular}

\section{6) Conocimiento respecto a la Hepatitis $B$ y verrugas genitales}

\begin{tabular}{|l|c|c|c|c|}
\hline Una vez curado el herpes ya no hay riesgo & 18 & 30.0 & 42 & 70.0 \\
\hline El uso de cremas espermicidas evita el contagio por clamidias & 14 & 23.3 & 46 & 76.7 \\
\hline La hepatitis B es una ITS & 28 & 46.7 & 32 & 53.3 \\
\hline Las vías de transmisión de Hepatitis B no son conocidas & 33 & 55.0 & 27 & 45.0 \\
\hline La Hepatitis B nunca deja secuelas & 14 & 23.4 & 46 & 76.6 \\
\hline Las verrugas genitales se contagian durante las relaciones & 50 & 83.3 & 10 & 16.7 \\
\hline Las verrugas genitales son genéticas & 17 & 28.3 & 43 & 71.7 \\
\hline
\end{tabular}

\section{7) Conocimiento sobre el VPH}

\begin{tabular}{|l|c|c|c|c|}
\hline El preservativo evita la transmisión de verrugas & 43 & 71.7 & 17 & 28.3 \\
\hline La infección por VPH puede ser por baños públicos & 32 & 53.3 & 28 & 46.7 \\
\hline El preservativo evita la transmisión de VPH & 44 & 73.3 & 16 & 26.7 \\
\hline La infección por VPH puede predisponer el cáncer & 41 & 68.3 & 19 & 31.7 \\
\hline Hay vacunas para las ITS & 35 & 58.3 & 25 & 41.7 \\
\hline Hay vacunas para el VPH & 53 & 88.3 & 7 & 11.7 \\
\hline Hay vacunas para el VIH & 30 & 50.0 & 30 & 50.0 \\
\hline
\end{tabular}

Nota: $f=$ Frecuencia; $\%=$ Porcentaje

$\boldsymbol{n}=60$

\section{REFERENCIAS BIBLIOGRÁFICAS}

1. Organización Mundial de la Salud (OMS). El embarazo en la adolescencia [internet]. OMS. 2018 [actualizado 23 febrero 2018, citado 19 octubre 2019]; Disponible en: https://www.who. int/es/news-room/fact-sheets/detail/adolescent-pregnancy

2. Fondo de las Naciones Unidas para la Infancia (UNICEF). La adolescencia: Una época de oportunidades [internet]. UNICEF. 2011 [citado 19 Octubre 2019]. Disponible en: https://www. unicef.org/bolivia/UNICEF___Estado_Mundial_de_la_Infancia_2011_-_La_adolescencia_una_epoca_de_oportunidades. pdf

3. León P, Minassian M, Borgoño R, Bustamante F. Embarazo adolescente. Rev Ped Elec [internet]. 2008 [citado 20 Octubre 2019]; 5(1), 42-51. Disponible en: http://www.enfermeriaaps. com/portal/download/SALUD\%20ADOLESCENTE/Embarazo\%20adolescente.pdf

4. Instituto Nacional de Estadística y Geografía (INEGI). Estrategia Nacional para la Prevención del Embarazo en Adolescentes [internet]. INEGI. 2018 [citado 25 Octubre 2019]. Disponible en: http://cedoc.inmujeres.gob.mx/documentos_download/ MHM_2018.pdf
5. Rosabal E, Romero N, Gaquín K, Hernández R. Conductas de riesgo en los adolescentes Revista Cubana de Medicina Militar [internet]. 2015 [citado 02 Noviembre 2019]; 44(2) 218-29. Disponible en: http://scielo.sld.cu/scielo.php?script=sci_abstract \&pid $=$ S0138-65572015000200010\&ln$\mathrm{g}=\mathrm{es} \& \mathrm{nrm}=\mathrm{iso}$

6. Favier MA, Samón M, Ruiz Y, Bonal AF. Factores de riesgos y consecuencias del embarazo en la adolescencia. Revista Información Científica [internet]. 2018 [citado 02 Noviembre 2019]; 97(1): 205-14. Disponible en: http://www. revinfcientifica.sld.cu/index.php/ric/article/view/1805/3517

7. Secretaria de Salud de Nuevo León (SSNL). Enfermedades de transmisión sexual [internet]. SSNL. 2015 [citado 03 Noviembre 2019]. Disponible en http://saludnl.gob.mx/ drupal/ enfermedades -de-transmisión-sexual

8. González DR, Pérez J, Sarduy M. Infección por el virus del papiloma humano en mujeres de edad mediana y factores asociados. Revista Cubana de Obstetricia y Ginecología [internet]. 2014 [citado 03 Noviembre 2019]; 40(2): 218-32. Disponible en: http://scielo.sld.cu/scielo. php?script=sci_ arttext\&pid=S0138-600X2014000200009 
9. Instituto Nacional de las Mujeres (INM). Estrategia Nacional para la Prevención del Embarazo en Adolescentes [internet]. INM. 2019 [actualizado 07 Enero 2019, citado 05 Noviembre 2019]. Disponible en: https://www.gob.mx/ inmujeres/acciones-y-programas/estrategia-nacional-para-la-prevencion-del-embarazo-en-adolescentes-33454

10. Vásquez APM, de Clavero AYS, Solaeche FML. Sexualidad en la Adolescencia. Estudio de grupos focales. Multiciencias [internet]. 2018 [citado 07 Noviembre 2019]; 17(1): 81-9. Disponible en: https://produccioncientificaluz.org/ index.php/multiciencias/article/ view/23616/23916

11. Actualidadrt [internet]. Organización de las Naciones Unidas para la Educación, la Ciencia y la Cultura (UNESCO). 2015 [actualizado 17 Junio 2015, citado 15 Noviembre 2019]. Disponible en: https://actualidad.rt.com/ rtpedia/177856-organizacion-educacion-ciencia-cultura-unesco

12.Montes DM, Gómez L. Socialización de la educación sexual en los estudiantes de la educación básica secundaria [tesis inédita de doctorado]. Universidad de la Costa [internet]. 2019; Disponible en: http://repositorio. cuc.edu.co/bitstream/handle/11323/5760/Socializaci\%C3 \%B3n\%20de\%20la\%20educaci\%C3\%B3n\%20sexual\%20 en\%20los\%20estudiantes\%20de\%20la\%20educaci\%C3\%B3n\%20b\%C3\%A1sica\%20secundaria\%20.pdf?sequence $=1 \&$ is Allowed $=\mathrm{y}$

13. Comisión Nacional de los Derechos Humanos (CNDH). NORMA Oficial Mexicana NOM-007-SSA2-2016; Para la atención de la mujer durante el embarazo, parto y puerperio, y de la persona recién nacida [internet]. CNDH. 2016 [actualizado 07 Abril 2016, citado 19 Noviembre 2019]. Disponible en https://www.cndh.org.mx/sites/all/ doc/Programas/NIH /Leyes\%20y\%20 normas\%20y\%20 reglamentos/Norma\%200ficial\%20Mexicana/NOM-007SSA2-2016\%20Embarazo,\%20parto\%20y\%20puerperio. pdf

14. Burns N, Grove SK, Gray J. Investigación en enfermería: desarrollo de la práctica enfermera basada en la evidencia. España: Elsevier. 2016. 580pp.

15. Grove SK, Gray JR, Faan PR. Investigación en enfermería: Desarrollo de la práctica enfermera basada en la evidencia. Elsevier. 2019. 520pp.

16. Barreto-Osorio RV, Campos MS, Carrillo-González GM, Coral-Ibarra R, Chaparro-Díaz L, Durán-Parra M, et al. Entrevista Percepción de Carga del Cuidado de Zarit: pruebas psicométricas para Colombia. Aquichan [internet]. 2015 [citado 25 Noviembre 2019]; 15(3): 368-80. Disponible en: http://dx.doi.org/10.5294/aqui.2015.15.3.5
17. Espada JP, Guillén-Riquelme A, Morales A, Orgilés M, Sierra JC. Validación de una escala de conocimiento sobre el $\mathrm{VIH}$ y otras infecciones de transmisión sexual en población adolescente. Atención Primaria [internet]. 2014 [citado 27 Noviembre 2019]; 46(10): 558-64. Disponible en: http://dx.doi.org/10.1016/j.aprim.2014.03.007

18. Secretaría de Salud (SS). Reglamento de la Ley General de Salud en Materia de Investigación para la Salud [internet]. SS. 2014 [actualizado 02 Abril 2014, citado 10 Octubre 2019]. Disponible en: http://www.diputados.gob.mx/LeyesBiblio/regley/ Reg_LGS_MIS.pdf

19. Rodríguez $A E$, Sucño CM. Factores que influyen en el embarazo en adolescentes en el centro de salud Pedro Sánchez Meza Chupaca [tesis inédita de bachillerato]. 2016 [citado 10 Diciembre 2019]; Disponible en: http://repositorio.upecen. edu.pe/bitstream/UPECEN/68/1/T102_47799237_T.pdf

20. Valenzuela MT, Ibarra R, María A, Zubarew G, Correa ML. Prevención de conductas de riesgo en el Adolescente: rol de familia [tesis inédita de bachillerato]. Index de Enfermería [internet]. 2013 [citado 10 Diciembre 2019]; 22(1-2): 50-4. Disponible en: http://dx.doi.org/10.4321/S1132-12962013000100011.

21. Silva-Fhon J, Andrade-Iraola K, Palacios-Vallejos E, Rojas-Huayta $V$, Mendonça-Júnior J. Nivel de conocimientos y comportamientos de riesgo relacionados con infecciones de transmisión sexual entre adolescentes mujeres. Enfermería universitaria [internet]. 2015 [citado 11 Diciembre 2019]; 11(4): 139-44. Disponible en: https://www.redalyc.org/ pdf/3587/358741841004.pdf

22. Contreras-González R, Magaly-Santana A, Jiménez-Torres E, Gallegos-Torres R, Xeque-Morales Á, Palomé-Vega G, Perea-Ortíz G. Nivel de conocimientos en adolescentes sobre el virus del papiloma humano. Enfermería universitaria [internet]. 2017 [citado 11 Diciembre 2019]; 14(2): 104-10. Disponible en: http://dx.doi.org/10.1016/j.reu.2017.01.002

23. Llanes HM, García D, Yedra M, Vargas Y. Intervención Educativa sobre infecciones de trasmisión sexual en adolescentes. Revista Cubana de Medicina General Integral [internet]. 2016 [citado 11 Diciembre 2019]; 32(4): 1-9. Disponible en: https:// doi.org/10.31434/rms.v4i4.202

24. Descriptores en Ciencias de la Salud. Descriptor: Enfermedades Transmisibles. [internet]. s/f. Disponible en:

http://decs.bvs.br/cgi-bin/wxis1660.exe/decsserver/?IsisScript $=$../cgi-bin/decsserver/decsserver.xis\&previous_page $=$ homepage\&task=exact_term\&interface_language $=$ e \& search_language =e\&search_exp=Enfermedades\%20 Transmisibles 
25. Descriptores en Ciencias de la Salud. Descriptor: Conocimiento [internet]. s/f. Disponible en:

http://decs.bvs.br/cgi-bin/wxis1660.exe/decsserver/?IsisScript $=$../cgi-bin/decsserver/decsserver.xis \&previous_page $=$ homepage\&task=exact_term\&interface_language $=e \&-$ search_language=e\&search_exp=Conocimiento

26. Descriptores en Ciencias de la Salud. Descriptor: Adolescente [internet]. s/f. Disponible en: http://decs.bvs.br/cgi-bin/ wxis1660.exe/decsserver/?IsisScript=../cgi-bin/decsserver/ decsserver.xis \&previous_page=homepage\&task=exact_term\&interface_language =e\&search_language =e\&search_ex$\mathrm{p}=$ Adolescente

27. Descriptores en Ciencias de la Salud. Descriptor: Embarazo en Adolescente [internet]. s/f. Disponible en: http://decs. bvs.br/cgi-bin/wxis1660.exe/decsserver/?lsisScript=../cgi-bin/ decsserver/decsserver.xis\&previous_page=homepage\&tas$k=$ exact_term\&interface_language $=e \&$ search_language $=e \&$ search_exp=Embarazo\%20en\%20Adolescencia

28. Gorrotxategi, P. Desconocimiento de las enfermedades de transmisión sexual en los adolescentes. Pediatría Atención Primaria [internet]. 2009 [citado 17 Junio 2020]; 11(41), 171-174. Disponible en: http://scielo.isciii.es/scielo.php?script=sci_arttext\&pid=S1139-76322009000100011 